Received: 5 January 2018

Accepted: 12 April 2018

Published online: 14 May 2018

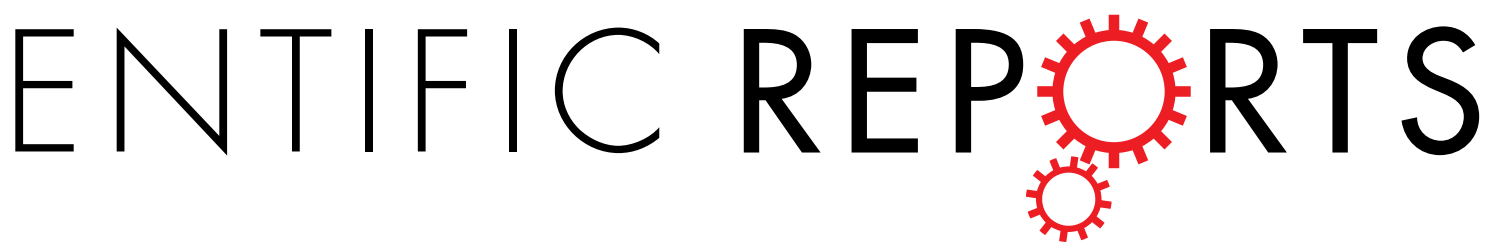

\title{
Aurora kinase A (AURKA) interaction with Wnt and Ras- MAPK signalling pathways in colorectal cancer
}

Annika Jacobsen ${ }^{1}$, Linda J. W. Bosch ${ }^{2}$, Sanne R. Martens-de Kemp ${ }^{2}$, Beatriz Carvalho ${ }^{2}$, Anke H. Sillars-Hardebol ${ }^{3}$, Richard J. Dobson ${ }^{4}{ }^{4}$, Emanuele de Rinaldis ${ }^{5}$, Gerrit A. Meijer ${ }^{2}$, Sanne Abeln $\mathbb{D}^{1}$, Jaap Heringa $\mathbb{( D}^{1}$, Remond J. A. Fijneman $\mathbb{D}^{2}$ \& K. Anton Feenstra $\mathbb{D}^{1}$

Hyperactivation of Wnt and Ras-MAPK signalling are common events in development of colorectal adenomas. Further progression from adenoma-to-carcinoma is frequently associated with $20 \mathrm{q}$ gain and overexpression of Aurora kinase A (AURKA). Interestingly, AURKA has been shown to further enhance Wnt and Ras-MAPK signalling. However, the molecular details of these interactions in driving colorectal carcinogenesis remain poorly understood. Here we first performed differential expression analysis (DEA) of AURKA knockdown in two colorectal cancer (CRC) cell lines with 20q gain and AURKA overexpression. Next, using an exact algorithm, Heinz, we computed the largest connected proteinprotein interaction (PPI) network module of significantly deregulated genes in the two CRC cell lines. The DEA and the Heinz analyses suggest $20 \mathrm{Wnt}$ and Ras-MAPK signalling genes being deregulated by AURKA, whereof $\beta$-catenin and KRAS occurred in both cell lines. Finally, shortest path analysis over the PPI network revealed eight 'connecting genes' between AURKA and these Wnt and Ras-MAPK signalling genes, of which UBE2D1, DICER1, CDK6 and RACGAP1 occurred in both cell lines. This study, first, confirms that AURKA influences deregulation of Wnt and Ras-MAPK signalling genes, and second, suggests mechanisms in CRC cell lines describing these interactions.

Colorectal cancer (CRC) is the third most common cancer in men and the second most common cancer in women worldwide ${ }^{1}$. In the early stages of CRC development, proliferative signalling is sustained by hyperactivation of the Wnt and Ras-MAPK signalling pathways due to mutations in key regulatory genes ${ }^{2}$. Disruption of Wnt signalling, caused by mutations in the APC tumour suppressor gene or other genes such as CTNNB1 (hereafter referred to as $\beta$-catenin), AXIN1 or AXIN2, promotes the progression from normal colon epithelium to a benign precursor lesion, called adenoma ${ }^{3}$. Subsequently, adenoma-to-carcinoma progression is driven by further genetic and epigenetic alterations. For example, in addition to the Ras-MAPK pathway, activated by mutation in e.g. the KRAS gene, other pathways important in carcinoma development are the TGF $\beta$ pathway, disrupted by mutation in e.g. SMAD4, and the TP53 pathway, disrupted by mutations in the TP53 gene ${ }^{4,5}$. Recently, it has been shown that adenoma organoids harbouring all these mutations can induce invasive cancers in mice only when a background of chromosomal instability is present ${ }^{6}$. This signifies the importance of chromosomal instability, which in fact occurs in $\sim 85 \%$ of $\mathrm{CRC}^{7}$, and is characterized by gross chromosomal aberrations.

Chromosomal arm $20 \mathrm{q}$ is frequently gained in $\mathrm{CRC}^{8,9}$ and has a strong association with the progression of colorectal adenoma to carcinoma ${ }^{10}$. Aurora kinase A (AURKA), a gene coding for a key cell cycle regulator, is located on $20 \mathrm{q}$. There is a significant correlation between the $20 \mathrm{q}$ copy number and increased AURKA mRNA

${ }^{1}$ Centre for Integrative Bioinformatics (IBIVU), Vrije Universiteit Amsterdam, Amsterdam, The Netherlands. ${ }^{2}$ Department of Pathology, The Netherlands Cancer Institute, Amsterdam, The Netherlands. ${ }^{3}$ Department of Pathology, VU University Medical Center, Amsterdam, The Netherlands. ${ }^{4}$ Bioinformatics group at the NIHR Biomedical Research Centre for Mental Health (IOP) and the South London and Maudsley NHS Trust, London, UK. 5The BRC Translational Bioinformatics Unit at the NIHR Biomedical Research Centre at Guy's and St Thomas' NHS Foundation Trust and King's College London, London, UK. Correspondence and requests for materials should be addressed to K.A.F. (email: k.a.feenstra@vu.nl) 
and protein expression ${ }^{11}$. Gain of $20 \mathrm{q}$ and/or AURKA overexpression is associated with a poor prognosis in many cancer types including $\mathrm{CRC}^{12-17}$.

AURKA overexpression has been shown to stabilize $\beta$-catenin levels and thereby activating Wnt signalling in gastric cancer cells by phosphorylating the negative regulator of $\beta$-catenin, GSK3B ${ }^{18,19}$. Also in glioma-initiating cells (distinguished by their capacity of self-renewal) AURKA is a negative regulator of $\beta$-catenin, by binding to AXIN $1^{20}$. Recently, it has been shown that AURKA upregulates Ras-MAPK signalling by interacting with the H-RAS/Raf-1 complex in kidney cells ${ }^{21}$. In addition, AURKA itself has been shown to be a target gene of both MAPK1/ERK2 signalling in pancreatic cancer cells ${ }^{22}$ and Wnt/ $\beta$-catenin signalling in multiple myeloma ${ }^{23}$. These data suggest a positive feedback loop from hyperactive proliferative signalling to AURKA overexpression, further inducing proliferative signalling cells ${ }^{21}$.

All this implies that there is interplay between AURKA and the Wnt and Ras-MAPK signalling pathways and vice versa in different cancer settings. For Wnt and Ras-MAPK signalling, much of the mechanisms have been elucidated, also in relation with $\mathrm{CRC}^{24}$, but such detail is not available for the interplay with AURKA. Although different molecular mechanisms are observed in the different settings this argues that the regulation itself is important. In this study, we used two distinct cell lines, SW480 and Caco2, both derived from colon carcinomas with 20q copy number gain and mutated TP53. However, the genetics differ between these cell lines, they originate from different individuals and therefore have different germline variations, and they have progressed to carcinomas independently and therefore also differ in their somatic alterations, such as DNA mutations and DNA copy number alterations. The distinct DNA copy number profiles of the two cell lines are shown in Supplementary Fig. S1. Further, a comparative study of colon cancer cell lines showed that out of five critical cancer genes (KRAS, BRAF, PIK3CA, PTEN, and TP53) only the mutation status of KRAS differed between the two cell lines (mutated in SW480). Further, of the other four genes TP53 was mutated in both cell lines ${ }^{25}$. Using SW480 and Caco2, we set out to investigate which key players and molecular interactions are involved in the interplay between AURKA and the Wnt and Ras-MAPK pathways that may drive progression of CRC.

\section{Results}

Differentially mRNA expression analysis. Differential expression analysis (DEA) was performed upon AURKA siRNA-directed downmodulation in two CRC cell lines with 20q gain and AURKA overexpression, SW480 and Caco2. AURKA was the most differentially downregulated gene in both cell lines (p-value of $1.69 \mathrm{e}-6$ in SW480 and 2.28e-7 in Caco2), indicating that the siRNA experiment was successful. The number of significantly expressed genes in response to AURKA downmodulation at a q-value less than 0.05 was 2,057 and 3,606 in SW480 and $\mathrm{Caco} 2$, respectively. 924 genes were significantly deregulated in both cell lines, whereof 50 were deregulated in opposite directions. In our analysis, however, we applied a more stringent threshold to determine significantly deregulated genes including both a q-value less than 0.05 and a fold change greater than 1.5 or less than -1.5 . This resulted in a 292 and 154 significantly deregulated genes in SW480 and Caco2, respectively. Of the 292 genes significantly deregulated in SW480, 139 genes were upregulated and 153 downregulated (Fig. 1A and Supplementary Table S1). Of the 154 genes significantly deregulated in Caco2, 73 genes were upregulated and 81 downregulated (Fig. 1B and Supplementary Table S2). Fifty-four genes were significantly deregulated in both cell lines (Fig. 1C and Table 1). Fifty-three of these 54 were deregulated in the same direction in both cell lines: 28 up in both and 25 down in both. The only gene deregulated in different directions was SLC12A2, which was up in Caco2 and down in SW480. These 53 genes are interesting candidates for the general influence of AURKA. GO enrichment analysis of these genes revealed that 'mitotic cell cycle' was the most significantly enriched process (Fig. 1D).

Identification of the most significantly deregulated gene modules. In order to gain understanding from the sets of genes differentially expressed by AURKA knockdown, we applied the Heinz algorithm ${ }^{26}$. Heinz integrates significant deregulation (the p-values from the DEA described above) with molecular protein interaction data (PPI network data from STRING) ${ }^{27}$. The algorithm looks for significantly deregulated gene modules (sets of genes connected in the PPI network), which might include genes that show no expression changes, based on the intuition that some regulatory effects may involve changes that are not visible at the expression level. The genes in these modules can therefore be considered 'functionally' deregulated. First, weights are assigned to each node reflecting its p-value from the DEA. The chosen FDR cut-off determines which nodes are assigned positive weights ( $p$-value below threshold) or negative (above threshold). The Heinz algorithm then computes the most significantly deregulated gene module, which is based both on the connectivity of the PPI network and the assigned weights. By thus combining the differential expression and protein interaction data, we can identify the most significantly deregulated gene modules (or functionally deregulated genes) upon AURKA downmodulation.

The FDR threshold was set to $6.21 \mathrm{e}-4$ for SW 480 and 2.40e-4 for Caco2. These thresholds were selected so that only the 50 most significantly deregulated genes were assigned positive weights. This selection allowed us to focus this analysis on gene modules of interpretable sizes (see Materials and Methods for further details). Figure 2 shows the most significantly deregulated gene modules for SW480 and Caco2. For SW480 the most significantly deregulated gene module consisted of 30 differentially expressed nodes (15 up and 15 down; see Fig. 2A and Supplementary Table S3). Twenty-three were significantly deregulated (positive weight, circles in Fig. 2A) and seven non-significant (negative weight, squares in Fig. 2A). For Caco2 the most significantly deregulated gene module consisted of 31 differentially expressed nodes (13 up and 18 down; see Fig. $2 \mathrm{~B}$ and Supplementary Table S4). Twenty-nine were significantly deregulated (positive weight, circles in Fig. 2B) and two non-significant (negative weight, square in Fig. 2B. Note that remaining genes out of the 50 genes with positive weights do not occur in the largest connected module, because they are not connected in the PPI network. The intersection between the most significantly deregulated gene modules of each of the two cell lines contains nine genes: AURKA, $\beta$-catenin, HMMR, KRAS, MBNL1, NUP98, REEP5, TNFRSF11B and UBE2D1 (black outline in nodes in Fig. 2A,B). These are all directly connected in the modules (bold edges in Fig. 2A,B), except for MBNL1, which 
A.

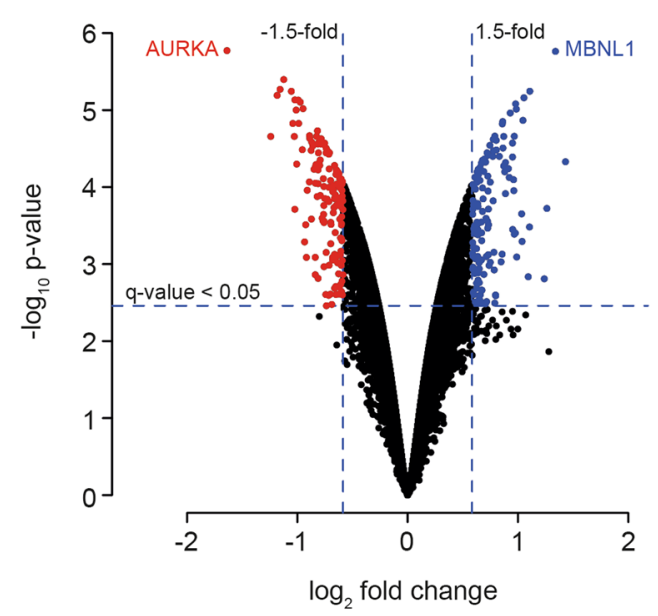

C.

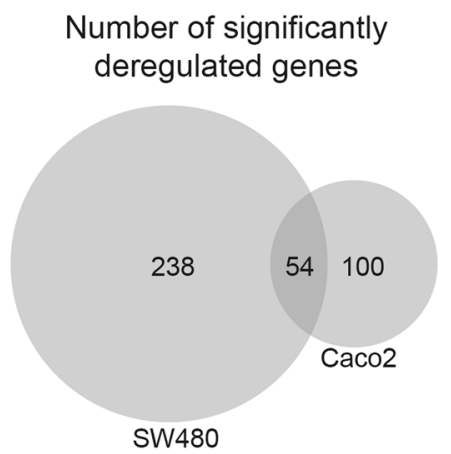

B.

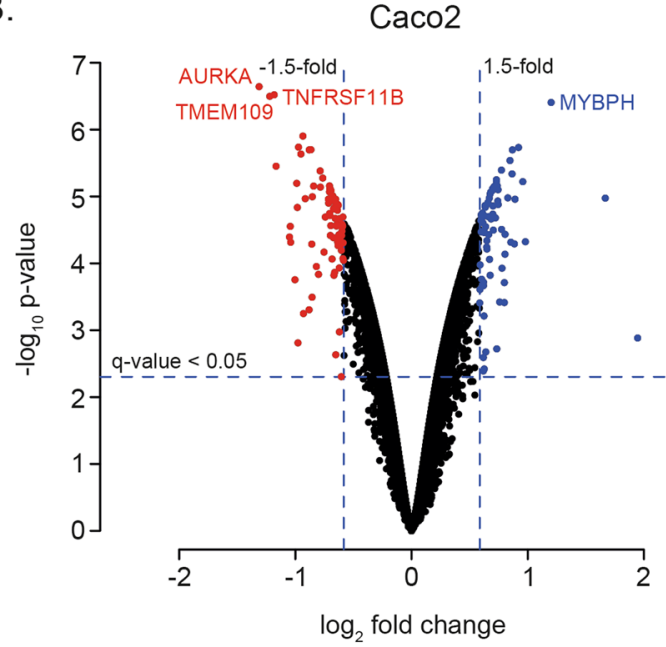

D.

\section{GO enrichment of intersection}

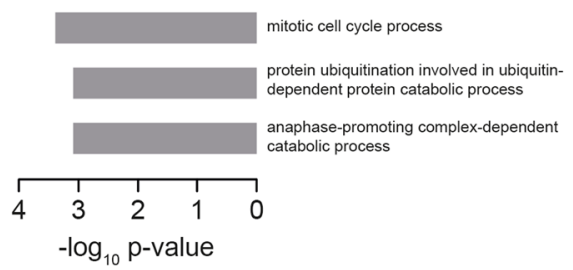

Figure 1. Differential mRNA expression analysis of AURKA knockdown in SW480 (A) and Caco2 (B) colorectal cancer cell lines. (A) and (B) Volcano plot of all unique genes from the differential mRNA expression analysis upon AURKA downmodulation for the SW480 and Caco2 cell lines, respectively. The vertical and horizontal lines illustrate the boundaries between significantly downregulated genes (red dots), significantly upregulated genes (blue dots) and non-significant differential genes (black dots). Horizontal lines represent the significance threshold of the q-value $<0.05$. Vertical lines represent the significance threshold of the fold change 1.5 ( $\log _{2}$ fold change 0.58$)$ up or down. C) VENN diagram of the number of significantly deregulated genes in SW480 and Caco2 (see Table 1). D) GO enrichment of the genes $(n=53)$ that are significantly deregulated in both cell lines in the same direction (the intersection in the Venn diagram in C minus one gene).

is connected via one intermediate node: in SW480 via JUN to KRAS, $\beta$-catenin and TNFRSF11B, and in Caco 2 via $A L B$ to KRAS, AURKA, $\beta$-catenin and TNFRSF11B, and via FOS to KRAS, UBE2D1, $\beta$-catenin and TNFRSF11B.

This integrative approach, using the p-values from the DEA and the PPI network data from STRING determined 30 and 31 genes in the most significantly deregulated gene modules for SW480 and Caco2, respectively. This analysis identified three genes for SW480 (JUN, RPS3A and LCK) and two genes for Caco2 ( $\beta$-catenin and FOS), which were not significantly deregulated in the DEA.

Proteins and interactions involved in enhanced Wnt and Ras-MAPK signalling by AURKA. We then set out to annotate Wnt and Ras-MAPK signalling genes that were either significantly deregulated in the gene-centric DEA or found in the most significantly deregulated gene modules in the integrative network-aware Heinz analysis. In total, 20 genes in either of these two signalling pathways were identified: four genes in the Wnt pathway (AXIN2, $\beta$-catenin, CTBP1 and WNT5A), eleven in the Ras-MAPK pathway (DDIT3, PDGFRA, RAP1B, KITLG, FOS, DUSP5, PLD1, KRAS, LAT, MAP3K6, RGL1), and five in both pathways (PRKCA, PPP3CA, RAC2, $P P P 3 R 1$ and $J U N)$ (see Fig. 3A). Two of these 20 deregulated genes, KRAS and $\beta$-catenin, appeared in both cell lines. The other 18 genes behaved cell line specific in these analyses: ten genes were found in SW480 only and eight genes were found only in Caco2 (Fig. 3A). When looking at Wnt and Ras-MAPK genes compared to all selected genes by the DEA (significantly deregulated) and the Heinz analysis (part of the most significantly deregulated gene module), respectively, we see different enrichments. For the DEA this was 11/292 (4\%) and 8/154 (5\%) for SW480 and Caco2, respectively (coloured nodes in Fig. 3A, and totals in Fig. 1C). On the other hand, for the Heinz analysis this was 5/30 (17\%) and 9/31 (29\%) for SW480 and Caco2, respectively (black dots vs. all nodes in Fig. 2A,B). Hence, Wnt and Ras-MAPK signalling genes were much more enriched in the deregulated modules selected by the network-aware Heinz analysis compared to the DEA, suggesting that their mutual interaction within the associated PPI network modules is important for CRC progression. 


\begin{tabular}{|c|c|c|c|c|c|c|c|}
\hline \multirow[b]{2}{*}{ Gene ID } & \multirow[b]{2}{*}{ Gene name } & \multicolumn{3}{|l|}{ SW480 } & \multicolumn{3}{|l|}{ Caco2 } \\
\hline & & p-value & q-value & FC** & p-value & q-value & FC** \\
\hline AURKA & Aurora kinase A & $1.69 \mathrm{e}-6$ & $1.86 \mathrm{e}-2$ & -1.64 & $2.28 \mathrm{e}-7$ & $1.96 \mathrm{e}-3$ & -1.31 \\
\hline REEP5 & Receptor accessory protein 5 & $2.20 \mathrm{e}-5$ & $1.86 \mathrm{e}-2$ & -1.24 & $1.83 \mathrm{e}-6$ & $5.18 \mathrm{e}-3$ & -0.97 \\
\hline SLC12A2 & Solute carrier family 12 member 2 & $5.70 e-6$ & $1.86 \mathrm{e}-2$ & -1.05 & $1.39 \mathrm{e}-5$ & $6.82 \mathrm{e}-3$ & 0.70 \\
\hline DICER1 & Dicer 1, ribonuclease III & $1.94 \mathrm{e}-4$ & $2.35 \mathrm{e}-2$ & -1.02 & $6.79 \mathrm{e}-5$ & $1.00 \mathrm{e}-2$ & -0.75 \\
\hline FEZ2 & Fasciculation and elongation protein zeta 2 & 7.41e-6 & $1.86 \mathrm{e}-2$ & -0.99 & 7.61e-6 & $6.82 \mathrm{e}-3$ & -0.70 \\
\hline KRAS & KRAS proto-oncogene, GTPase & $9.58 \mathrm{e}-6$ & $1.86 \mathrm{e}-2$ & -0.95 & $1.10 \mathrm{e}-5$ & $6.82 e-3$ & -0.65 \\
\hline UBE2D1 & Ubiquitin conjugating enzyme E2 D1 & $3.08 \mathrm{e}-4$ & $2.55 \mathrm{e}-2$ & -0.92 & $1.08 \mathrm{e}-5$ & $6.82 e-3$ & -0.91 \\
\hline TM4SF1 & Transmembrane $4 \mathrm{~L}$ six family member 1 & $1.10 \mathrm{e}-4$ & $2.28 \mathrm{e}-2$ & -0.91 & $2.03 e-5$ & $7.98 \mathrm{e}-3$ & -0.74 \\
\hline ACVR1 & Activin A receptor type 1 & $2.60 \mathrm{e}-4$ & $2.46 \mathrm{e}-2$ & -0.87 & $6.36 \mathrm{e}-6$ & $6.82 \mathrm{e}-3$ & -0.99 \\
\hline MARVELD2 & MARVEL domain containing 2 & $2.61 e-5$ & $1.86 \mathrm{e}-2$ & -0.87 & $2.13 e-5$ & $7.98 \mathrm{e}-3$ & -0.66 \\
\hline BORCS8 & BLOC-1-related complex subunit 8 & $2.91 \mathrm{e}-5$ & $1.86 \mathrm{e}-2$ & -0.86 & $1.61 \mathrm{e}-5$ & $7.31 \mathrm{e}-3$ & -0.64 \\
\hline SLC25A24 & Solute carrier family 25 member 24 & $5.87 \mathrm{e}-5$ & $2.10 \mathrm{e}-2$ & -0.85 & $1.10 \mathrm{e}-5$ & $6.82 \mathrm{e}-3$ & -0.71 \\
\hline MBOAT2 & $\begin{array}{l}\text { Membrane bound O-acyltransferase domain } \\
\text { containing } 2\end{array}$ & $1.73 e-4$ & $2.35 \mathrm{e}-2$ & -0.76 & $7.00 \mathrm{e}-6$ & $6.82 \mathrm{e}-3$ & -0.84 \\
\hline ACOX2 & Acyl-CoA oxidase 2 & $3.59 \mathrm{e}-5$ & $1.86 \mathrm{e}-2$ & -0.74 & $2.78 \mathrm{e}-5$ & $8.77 e-3$ & -0.61 \\
\hline TNFRSF11B & TNF receptor superfamily member $11 \mathrm{~b}$ & $3.13 e-5$ & $1.86 \mathrm{e}-2$ & -0.74 & $3.02 \mathrm{e}-7$ & $1.96 \mathrm{e}-3$ & -1.18 \\
\hline $\mathrm{NA}^{*}$ & ENSG00000260912* & $3.47 \mathrm{e}-3$ & $4.91 \mathrm{e}-2$ & -0.74 & $4.04 \mathrm{e}-5$ & $9.30 \mathrm{e}-3$ & -1.05 \\
\hline NA* & ENSG00000234119* & $3.74 \mathrm{e}-5$ & $1.91 \mathrm{e}-2$ & -0.71 & $8.20 \mathrm{e}-6$ & $6.82 \mathrm{e}-3$ & -0.69 \\
\hline SNX24 & Sorting nexin 24 & $2.46 \mathrm{e}-3$ & $4.32 \mathrm{e}-2$ & -0.70 & $5.16 e-5$ & $9.83 e-3$ & -0.86 \\
\hline ANP32 A & $\begin{array}{l}\text { Acidic nuclear phosphoprotein } 32 \text { family } \\
\text { member A }\end{array}$ & $8.22 \mathrm{e}-5$ & $2.26 \mathrm{e}-2$ & -0.70 & $2.15 e-5$ & $7.98 \mathrm{e}-3$ & -0.62 \\
\hline CANX & Calnexin & $5.44 \mathrm{e}-4$ & $2.89 \mathrm{e}-2$ & -0.70 & $2.32 \mathrm{e}-6$ & $5.52 e-3$ & -0.95 \\
\hline TMX2P1 & $\begin{array}{l}\text { Thioredoxin related transmembrane protein } 2 \\
\text { pseudogene } 1\end{array}$ & $2.87 \mathrm{e}-4$ & $2.49 \mathrm{e}-2$ & -0.70 & $5.42 \mathrm{e}-5$ & $9.96 e-3$ & -0.63 \\
\hline B4GALT4 & Beta-1,4-galactosyltransferase 4 & $8.17 \mathrm{e}-4$ & $3.19 \mathrm{e}-2$ & -0.68 & $3.53 e-6$ & $6.82 \mathrm{e}-3$ & -1.17 \\
\hline SNX4 & Sorting Nexin 4 & $1.09 \mathrm{e}-4$ & $2.28 \mathrm{e}-2$ & -0.67 & $4.74 \mathrm{e}-5$ & $9.65 e-3$ & -0.61 \\
\hline TSPYL4 & TSPY like 4 & $1.41 \mathrm{e}-4$ & $2.35 \mathrm{e}-2$ & -0.66 & $8.55 \mathrm{e}-6$ & $6.82 \mathrm{e}-3$ & -0.71 \\
\hline PHLDA2 & $\begin{array}{l}\text { Pleckstrin homology like domain family A } \\
\text { member } 2\end{array}$ & $1.67 \mathrm{e}-3$ & $3.74 \mathrm{e}-2$ & -0.60 & $4.19 \mathrm{e}-5$ & $9.37 e-3$ & -0.61 \\
\hline TP53I3 & Tumor protein p53 inducible protein 3 & $1.31 \mathrm{e}-4$ & $2.35 \mathrm{e}-2$ & -0.59 & $2.61 \mathrm{e}-5$ & $8.77 \mathrm{e}-3$ & -0.60 \\
\hline ARHGAP19 & Rho GTPase activating protein 19 & $2.25 \mathrm{e}-4$ & $2.37 \mathrm{e}-2$ & 0.59 & $7.93 e-6$ & $6.82 \mathrm{e}-3$ & 0.74 \\
\hline LAMC1 & Laminin subunit gamma 1 & $6.03 \mathrm{e}-4$ & $2.95 \mathrm{e}-2$ & 0.60 & $6.01 \mathrm{e}-6$ & $6.82 \mathrm{e}-3$ & 0.96 \\
\hline STK17B & Serine/threonine kinase 17b & $1.32 \mathrm{e}-3$ & $3.54 \mathrm{e}-2$ & 0.61 & $5.71 e-5$ & $1.00 \mathrm{e}-2$ & 0.65 \\
\hline NRP2 & Neuropilin 2 & $2.83 e-3$ & $4.55 \mathrm{e}-2$ & 0.65 & $4.83 e-5$ & $9.65 \mathrm{e}-3$ & 0.85 \\
\hline IDNK & IDNK, gluconokinase & $2.18 \mathrm{e}-3$ & $4.17 \mathrm{e}-2$ & 0.67 & $2.16 \mathrm{e}-4$ & $1.34 \mathrm{e}-2$ & 0.62 \\
\hline KTN1 & Kinetin 1 & $4.50 \mathrm{e}-5$ & $2.05 \mathrm{e}-2$ & 0.68 & $1.88 \mathrm{e}-5$ & $7.93 e-3$ & 0.60 \\
\hline RACGAP1 & Rac GTPase activating protein 1 & $2.77 \mathrm{e}-4$ & $2.49 \mathrm{e}-2$ & 0.72 & $6.11 \mathrm{e}-5$ & $1.00 \mathrm{e}-2$ & 0.65 \\
\hline CDK6 & Cyclin dependent kinase 6 & $8.63 e-5$ & $2.26 \mathrm{e}-2$ & 0.75 & $4.60 \mathrm{e}-6$ & $6.82 \mathrm{e}-3$ & 0.86 \\
\hline SPA17 & Sperm autoantigenic protein 17 & $1.51 \mathrm{e}-4$ & $2.35 \mathrm{e}-2$ & 0.77 & $2.00 \mathrm{e}-5$ & $7.98 \mathrm{e}-3$ & 0.66 \\
\hline LINC00467 & Long intergenic non-protein coding RNA 467 & $3.30 \mathrm{e}-5$ & $1.86 \mathrm{e}-2$ & 0.80 & $8.62 \mathrm{e}-6$ & $6.82 \mathrm{e}-3$ & 0.68 \\
\hline BUB1B & $\begin{array}{l}\text { BUB1 mitotic checkpoint serine/threonine } \\
\text { kinase B }\end{array}$ & $3.99 \mathrm{e}-5$ & $1.94 \mathrm{e}-2$ & 0.83 & $1.84 \mathrm{e}-5$ & $7.89 \mathrm{e}-3$ & 0.70 \\
\hline BIK & BCL2 interacting killer & $3.11 \mathrm{e}-5$ & $1.86 \mathrm{e}-2$ & 0.86 & $2.01 \mathrm{e}-6$ & $5.18 \mathrm{e}-3$ & 0.87 \\
\hline SH3D19 & SH3 domain containing 19 & $1.22 \mathrm{e}-4$ & $2.35 \mathrm{e}-2$ & 0.89 & $1.05 e-5$ & $6.82 \mathrm{e}-3$ & 0.83 \\
\hline EIF4EBP2 & $\begin{array}{l}\text { Eukaryotic translation initiation factor } 4 \mathrm{E} \\
\text { protein binding } 2\end{array}$ & $7.50 e-5$ & $2.26 \mathrm{e}-2$ & 0.92 & $2.09 e-5$ & $7.98 \mathrm{e}-3$ & 0.72 \\
\hline HMMR & Hyaluronan mediated motility receptor & $4.07 \mathrm{e}-5$ & $1.95 \mathrm{e}-2$ & 0.95 & $1.17 \mathrm{e}-5$ & $6.82 \mathrm{e}-3$ & 0.74 \\
\hline KLHL15 & Kelch like family member 15 & $3.55 e-4$ & $2.64 \mathrm{e}-2$ & 0.95 & $2.46 \mathrm{e}-4$ & $1.42 \mathrm{e}-2$ & 0.59 \\
\hline DYRK2 & $\begin{array}{l}\text { Dual specificity tyrosine phosphorylation } \\
\text { regulated kinase } 2\end{array}$ & $4.09 \mathrm{e}-4$ & $2.69 \mathrm{e}-2$ & 0.97 & $3.68 \mathrm{e}-5$ & $9.30 \mathrm{e}-3$ & 0.80 \\
\hline ZNF268 & Zink finger protein 268 & $3.95 e-4$ & $2.66 \mathrm{e}-2$ & 0.98 & $8.72 \mathrm{e}-5$ & $1.07 \mathrm{e}-2$ & 0.67 \\
\hline NUP98 & Nucleoporin 98 & $9.71 \mathrm{e}-6$ & $1.86 \mathrm{e}-2$ & 0.98 & $6.76 \mathrm{e}-6$ & $6.82 \mathrm{e}-3$ & 0.73 \\
\hline MTMR6 & Myotubularin related protein 6 & $8.09 \mathrm{e}-4$ & $3.19 \mathrm{e}-2$ & 0.99 & $1.18 \mathrm{e}-4$ & $1.20 \mathrm{e}-2$ & 0.78 \\
\hline PTP4A1 & $\begin{array}{l}\text { Protein tyrosine phosphatase type IVA, } \\
\text { member } 1\end{array}$ & $5.08 \mathrm{e}-4$ & $2.85 \mathrm{e}-2$ & 1.04 & $1.94 \mathrm{e}-4$ & $1.31 \mathrm{e}-2$ & 0.80 \\
\hline GOLT1B & Golgi transport 1B & $1.36 \mathrm{e}-5$ & $1.86 \mathrm{e}-2$ & 1.04 & $1.95 \mathrm{e}-5$ & $7.98 \mathrm{e}-3$ & 0.63 \\
\hline RPS27L & Ribosomal protein S27 like & 6.91e-6 & $1.86 \mathrm{e}-2$ & 1.05 & $1.06 \mathrm{e}-5$ & $6.82 \mathrm{e}-3$ & 0.67 \\
\hline MALAT1 & $\begin{array}{l}\text { Metastasis associated lung adenocarcinoma } \\
\text { transcript } 1\end{array}$ & $1.46 \mathrm{e}-3$ & $3.66 \mathrm{e}-2$ & 1.09 & $1.06 e-5$ & $6.82 \mathrm{e}-3$ & 1.67 \\
\hline & & & & & & & \\
\hline
\end{tabular}




\begin{tabular}{|c|c|c|c|c|c|c|c|}
\hline \multirow[b]{2}{*}{ Gene ID } & \multirow[b]{2}{*}{ Gene name } & \multicolumn{3}{|l|}{ SW480 } & \multicolumn{3}{|l|}{ Caco2 } \\
\hline & & p-value & q-value & FC** & p-value & q-value & FC** \\
\hline LAMP2 & Lysosomal associated membrane protein 2 & $3.30 \mathrm{e}-4$ & $2.62 \mathrm{e}-2$ & 1.10 & $4.75 e-5$ & $9.65 e-3$ & 0.98 \\
\hline SYPL1 & Synaptophysin like 1 & $5.69 \mathrm{e}-6$ & $1.86 \mathrm{e}-2$ & 1.11 & $5.63 e-6$ & $6.82 \mathrm{e}-3$ & 0.73 \\
\hline ARRDC4 & Arrestin domain containing 4 & $1.55 \mathrm{e}-3$ & $3.70 \mathrm{e}-2$ & 1.24 & $2.11 \mathrm{e}-3$ & $3.15 \mathrm{e}-2$ & 0.64 \\
\hline MBNL1 & Muscleblind like splicing regulator 1 & $1.73 e-6$ & $1.86 \mathrm{e}-2$ & 1.34 & $5.91 \mathrm{e}-6$ & $6.82 \mathrm{e}-3$ & 0.73 \\
\hline
\end{tabular}

Table 1. Genes significantly deregulated in both the Caco 2 and SW480 cell lines under AURKA knockdown $(\mathrm{n}=54)$. $\mathrm{p}$-value, $\mathrm{q}$-value and $\log _{2}$ fold change $(\mathrm{FC})$ from the two differential mRNA expression analyses are shown for each gene ID. The Gene names are retrieved from Ensembl. The table is sorted on $\mathrm{SW} 480 \log _{2} \mathrm{FC}$. *Gene id and gene name are not available. The Ensembl id is provided as identifier. $* * F C=\log _{2}$ fold change.

A.

SW480
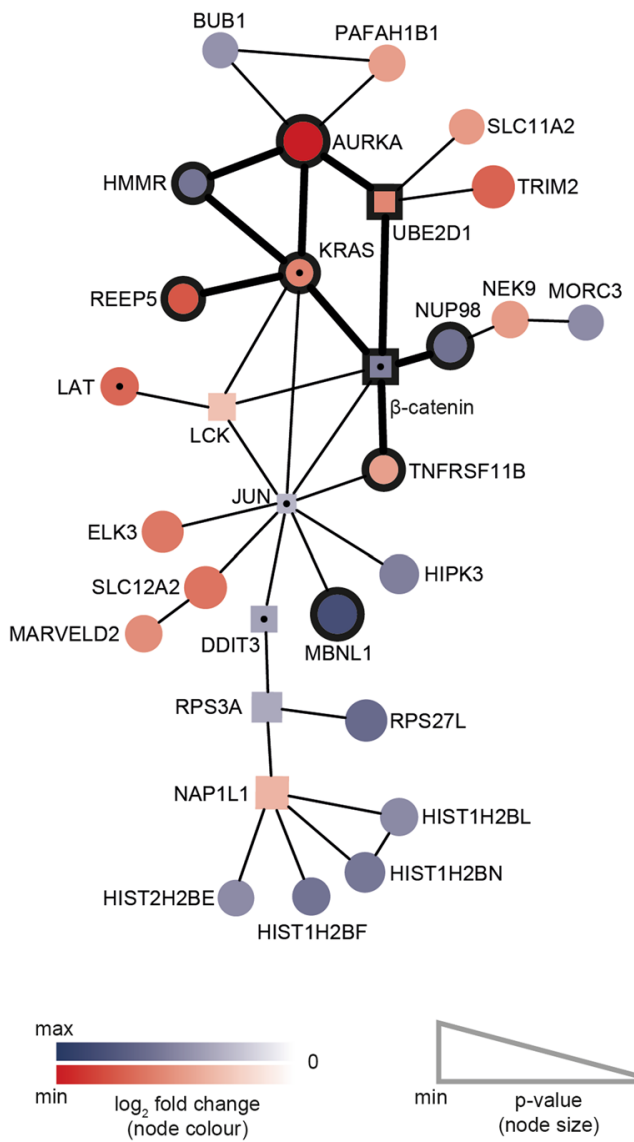

B.

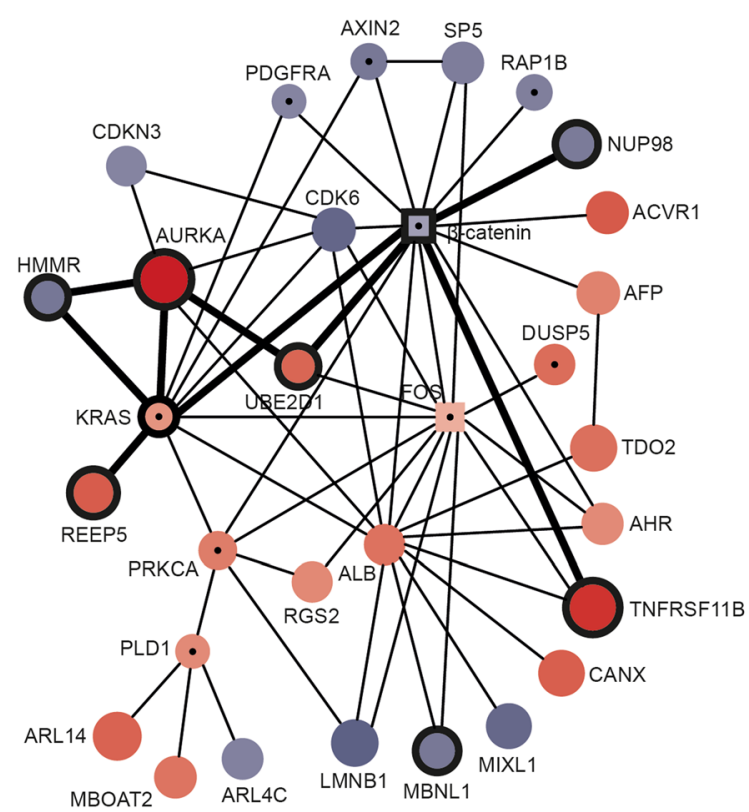

square nodes are non-significant

round nodes are significant

- nodes with a dot are in the Wnt and/or Ras-MAPK pathway

Figure 2. Significantly deregulated gene modules in SW480 (A) and Caco2 (B) derived from integrating p-values from differential mRNA expression analysis (DEA) upon downmodulation of AURKA and STRING protein-protein interaction network data using the Heinz algorithm. (A) and (B) Based on the chosen FDR threshold of 6.21e-4 for SW480 and 2.40e-4 for Caco2 in the Heinz analysis, round nodes are significant (p-value lower than the FDR threshold), and squared nodes are non-significant (p-value above the FDR threshold). The size of the nodes is based on the p-values from the DEA: a lower p-value corresponds to a bigger node. The colour of the nodes indicates the direction of the fold change with respect to AURKA downmodulation in the DEA. Red nodes are downregulated and blue nodes upregulated. The colour intensity of the nodes is correlated with the magnitude of the fold change, where the greatest intensities represent the highest absolute fold change. The nodes of the nine genes occurring in both modules are outlined in black and the direct edges between these are in bold. The nodes representing proteins in the Wnt or Ras-MAPK pathways have a black dot.

The most significantly deregulated gene modules also suggest interactions between AURKA and the Wnt and Ras-MAPK pathway (Fig. 2). Only KRAS is directly connected to AURKA, whereas the other genes are indirectly connected to AURKA via one or more genes. In order to identify interactions between AURKA and the remaining genes of the $20 \mathrm{Wnt}$ and Ras-MAPK genes, we applied a shortest path analysis through the protein 


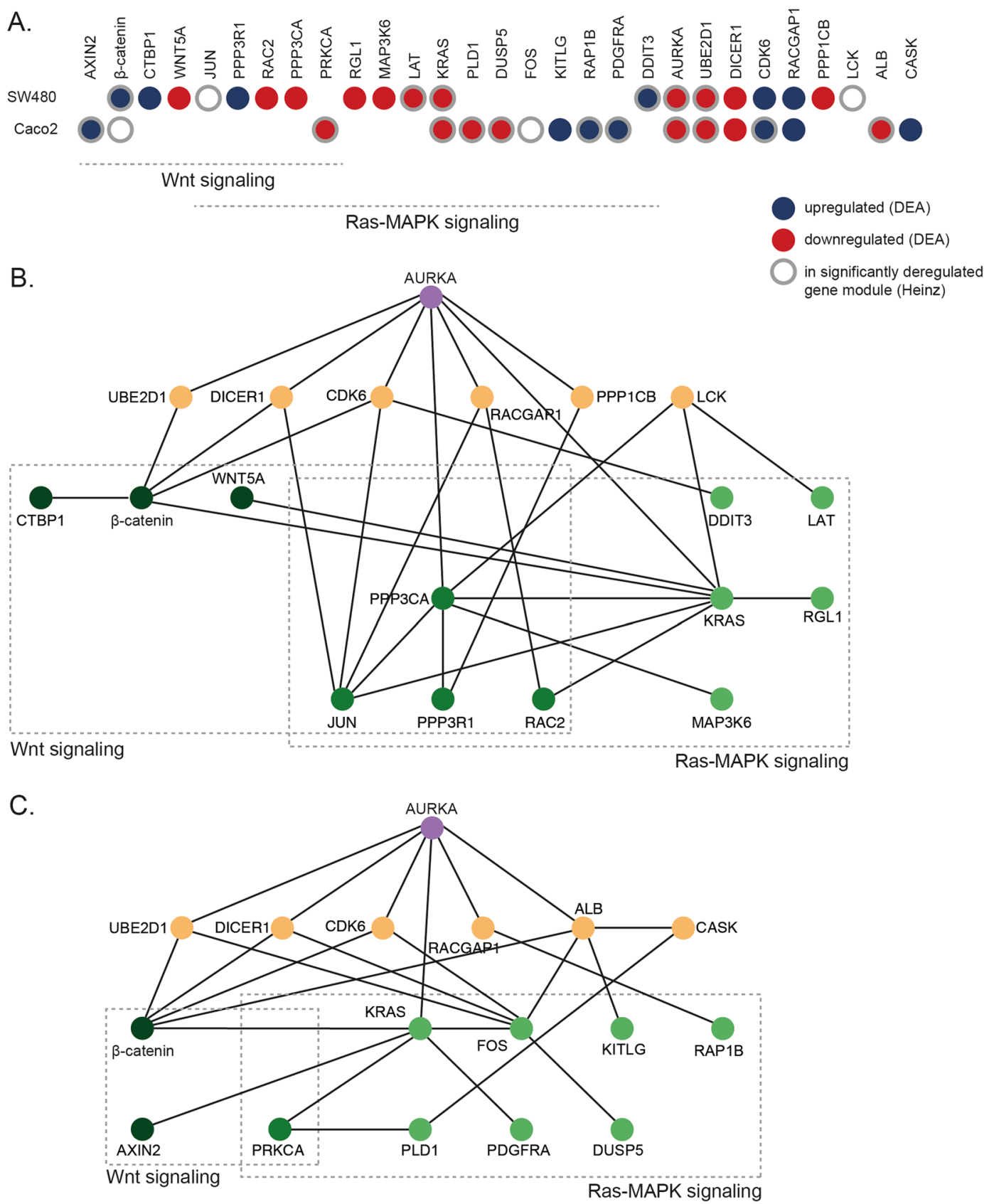

Figure 3. Wnt and Ras-MAPK pathway genes and interacting genes with AURKA, significantly deregulated (DEA) or in the most significantly deregulated gene module (Heinz) in the two cell lines, SW480 and Caco2. (A) Overview of AURKA, the genes in the Wnt and Ras-MAPK pathways, and the genes connecting these, either significantly deregulated (DEA; q-value $<0.05$, red for down and blue for upregulation) or found in the most significantly deregulated gene module (Heinz, grey edges) in the two cell lines. (B) and (C) Interactions between AURKA (top-middle, purple) and the Wnt and Ras-MAPK signalling genes (green shades) deduced together with additional 'connecting genes' (orange) using a shortest path analysis in SW480 (B) and Caco2 (C).

interaction network. This analysis was repeated for each of the cell lines, SW480 and Caco2. We used a subset of the STRING PPI only consisting of the genes that were either significantly deregulated in the DEA or part of the most significantly deregulated module from the Heinz analysis. The shortest path between each of these genes to AURKA was determined for SW480 (Fig. 3B) and Caco2 (Fig. 3C). Some of these interactions are directly from AURKA to Wnt and Ras-MAPK genes, but most are indirectly via additional interactions outside Wnt or Ras-MAPK signalling. For both cell lines there were six such external 'connecting genes': LCK and PPP1CB in SW480, and $A L B$ and CASK in Caco2, and four shared: UBE2D1, DICER1, CDK6 and RACGAP1. We propose that these genes could be important to explain the observed correlation between AURKA modulation and Wnt and Ras-MAPK activity. 
TCGA CRC data analysis. The Wnt and Ras-MAPK pathway proteins and their molecular interactions with AURKA identified in this study are based on two CRC cell lines with 20q gain where AURKA is downmodulated. These results provide us with a better understanding of possible interconnections between AURKA and Wnt and Ras-MAPK signalling, however, to gain insight into the CRC progression it is necessary to study these genes from a cellular context that better represents the physiological environment. In CRC tissue samples where epithelial neoplastic cells interact with their tumour microenvironment the biology is more complex and heterogeneous than in isolated epithelial cancer cell lines.

We analysed publicly available CRC samples from TCGA (RNA-seq and somatic copy number aberration data) to see if we could recover the significantly deregulated genes from the CRC cell line DEA. Out of 330 microsatellite stable tumour samples, $217(65.76 \%)$ had AURKA copy number aberration, determined by a segment mean threshold of 0.4 in the somatic copy number aberration data (see Materials and Methods). DEA was performed between the AURKA-no-gain and AURKA-gain samples using the RNA-seq expression data. Note this atypical order of the sample groups (no-gain vs. gain instead of gain vs. no-gain) is to ensure proper comparison to the distinctive setup of the cell line experiments where AURKA is downmodulated (see Materials and Methods). AURKA was significantly deregulated ( $\mathrm{p}$-value $=1.62 \mathrm{e}-26)$, demonstrating the correlation between increased AURKA count and copy number gain. In total, 1308 genes were significantly deregulated in the AURKA no-gain samples with a significance threshold of the adjusted p-value less than $10^{-5}, 751$ up and 557 down (Supplementary Table S6). We then investigated the significance and directionality of the genes located on 20q. First, we retrieved the Ensembl ids for the genes located on 20q11-20q13.33 from BioMart. Second, we mapped these Ensembl ids to the Ensembl ids in the TCGA samples, which resulted in 316 genes. Out of these, 168 were significantly deregulated, 164 down and 4 up. Thus, 163 genes located on 20q11-20q13.33 are significantly deregulated in the same direction as AURKA (Supplementary Table S7).

We compared the significantly deregulated genes in the TCGA AURKA no-gain samples $(\mathrm{n}=1308)$ to the sets of genes significantly deregulated in the SW480 $(n=292)$ and Caco2 $(n=154)$ DEA (described above). A minor overlap was found; only 24 genes were significantly deregulated in the TCGA and either of the cell lines (14 in SW480 and 13 in Caco2). Further, the majority of these genes were deregulated in opposite directions between the two different settings (see Supplementary Fig. S4).

\section{Discussion}

In this study we identified 20 Wnt and Ras-MAPK signalling genes and eight additional connecting genes that suggest possible mechanisms of interaction between AURKA and Wnt and Ras-MAPK signalling in a CRC cell line setting. We performed mRNA expression analysis on AURKA knockdown in two CRC cell lines, SW480 and $\mathrm{Caco} 2$, and applied three main computational approaches to interpret this expression data. First, we applied straightforward gene-centric DEA determining significantly deregulated genes. Second, we applied the Heinz algorithm, an integrative network-aware analysis, using the expression data and the STRING PPI network data to determine significantly deregulated gene modules. Third, we applied a shortest path analysis determining connections between AURKA and Wnt and Ras-MAPK signalling genes that were significantly deregulated in the first two analyses, DEA and Heinz. The DEA resulted in the largest set of genes, however it does not by itself reveal how their deregulation may be intertwined. The Heinz integrative analysis clearly revealed in an unbiased way the involvement of the Wnt and Ras-MAPK pathways, as well as indicated some plausible mechanisms of interaction between AURKA and both pathways. Finally, the shortest path analysis added 8 connecting genes that may be relevant to explain how AURKA modulation may affect the activity of these pathways, and thereby further induce proliferation in developing carcinoma cells.

In previous studies interactions between AURKA and Wnt signalling have been observed in gastric cancer cell lines ${ }^{18,19}$ and glioma-initiating cells ${ }^{20}$, and interactions between AURKA and Ras-MAPK signalling have been observed in kidney cells $s^{21}$, pancreatic cancer cells ${ }^{22}$ and multiple myeloma ${ }^{23}$. Thus the link between AURKA and Wnt and Ras-MAPK signalling is not new in general, but the specific proteins that are deregulated differ between the different cellular contexts. For instance, GSK3B and AXIN1 are two proteins deregulated by AURKA leading to stimulation of Wnt signalling in gastric cancer cell lines ${ }^{18,19}$ and glioma-initiating cells ${ }^{20}$, respectively. Activation of Ras-MAPK by AURKA has been attributed to interaction between AURKA and the H-RAS/Raf-1 complex in kidney cells ${ }^{21}$. In our analysis, in the two CRC cell lines, SW480 and Caco2, differential expression of GSK3B, AXIN1 and HRAS, was not recovered, however, AXIN2 and KRAS, homologous of AXIN1 and HRAS, respectively, were recovered. Besides the differences in the cellular contexts, a plausible explanation for not recovering GSK3B and AXIN1 in our analysis could be that GSK3B phosphorylation and AXIN1 binding to AURKA and $\beta$-catenin involve changes at the protein level, which would not be visible in our mRNA expression data. Another limitation of our experimental setup is that $A U R K A$ is the only gene that is downmodulated, whereas in an in vivo setting more genes on $20 \mathrm{q}$ are deregulated together with AURKA. One example is TPX2, which just as with AURKA is located on $20 \mathrm{q}$, and implied to be involved with gain of $20 \mathrm{q}^{11}$, however, in our analysis TPX2 is not modulated.

Based on our analysis, the genes in the Wnt and Ras-MAPK pathways affected by AURKA downmodulation were mostly unique per cell line. In the Wnt pathway, only $\beta$-catenin was affected in both cell lines, and in the Ras-MAPK pathway, it was only KRAS. It should be noted, however, that these genes are at the core of their respective pathways, which suggests that in both cellular contexts, perturbing these pathways may be very important. The molecular differences can be explained by the cause or the effect of the perturbations being dependent on the particular cellular context. On the other hand, the 'connecting genes' (connecting AURKA to the Wnt and Ras-MAPK signalling genes) were mostly similar between the cell lines (four out of six genes shared in both). While both SW480 and Caco2 are CRC cell lines with 20q gain, AURKA overexpression, and TP53 mutation, they also show differences. Firstly, the cell lines originate from different individuals, and therefore different germline variations. Secondly, the two cell lines have progressed to carcinomas independently. One specific difference is 
that KRAS is mutated in SW480 but not in $\mathrm{Caco}^{25}$. However, their genetic differences are much more extensive than this (see DNA copy number profiles in Supplementary Fig. S1). Consequently, the underlying molecular biology of SW480 and Caco2 differs and they are therefore expected to respond differently to a perturbation such as AURKA downmodulation when looking at the expression of individual genes, although the overall changes in pathways could still be the same. For instance, KRAS is one of the most connected genes in both cell lines in our two network aware analyses (Figs 2 and 3), while at the same time the differentially expressed networks upon AURKA knockdown show different genes connected to KRAS in both cell lines. This could potentially be due to the different mutation status of KRAS between the cell lines. For instance, in the shortest path analysis (Fig. 3) KRAS is connected to AURKA and $\beta$-catenin in both cell lines. However, in SW480, where KRAS is mutated, KRAS in addition is connected to LCK, WNT5A, PPP3CA, JUN, and RAC2. Whereas, in Caco2, KRAS is in addition connected to FOS, AXIN2, and PRKCA.

The four consensus molecular subtypes (CMS) of colorectal cancer distinguish differences in signalling activities $^{28}$. CMS2 and CMS4 are the most common chromosomal instable subtypes, both containing 20q gain, with CMS2 having a better prognosis than CMS4. CMS2 show increased proliferation, whereas CMS4 exhibit invasion. In a recent $s t u d y^{29}$, they found that four genes were sufficient to predict CMS4: PDGFRA, PDGFRB, $P D G F C$ and KIT. Interestingly, in the current study, PDGFRA (p-value $=1.28 \mathrm{e}-05)$ and the KIT ligand, KITLG ( $\mathrm{p}$-value $=3.19 \mathrm{e}-05)$, were significantly deregulated in Caco2, but not in SW480 (possibly explained by the different genetic backgrounds). Thus upon AURKA downmodulation Caco2 resembles the CMS4 subtype, and it can be implied that Caco2 also displays the CMS4 phenotype, invasion.

The cell line results presented in this paper suggest possible interconnections between AURKA and Wnt and Ras-MAPK signalling. Analysis of the CRC tissue samples from the TCGA data provided results from a more appropriate cellular context for studying CRC progression. There was an overlap of 24 genes when comparing the significantly deregulated genes from the TCGA analysis and the cell line analyses. However, most of these overlapping significantly deregulated genes were deregulated in opposite directions between the two different settings (Supplementary Fig. S4). We should here take into account the big differences between these two settings. First, in the tissue samples from TCGA $A U R K A$ is gained as a result of gain in $20 \mathrm{q}$, on which many more genes than only AURKA are located and affected (Supplementary Table S7). In the cell line experiments, however, only AURKA is downmodulated, whereas all the other genes on $20 \mathrm{q}$ have remained unchanged. Second, in a tumor, epithelial neoplastic cells interact with their tumor microenvironment. Thus, the tissue samples are much more complex than the cell lines, and most likely there would be feedback mechanisms active in this environment that do not operate in the isolated cell lines. Third, a tumor is very heterogeneous, whereas a cell line is much more homogeneous. Thus, the presence of multiple clones within a tumor might also mask the effect of AURKA gain present in a minority of the clones in the tumor. Thus, we believe that because of these big differences between the cell line and tissue settings, a gene that is deregulated in both cases does not necessarily have to be deregulated in the same direction, but the overlap of deregulated genes, and their pathways, provides a good starting point for further investigation of the mechanisms between AURKA and Wnt and Ras-MAPK signalling. At the same time, this also underlines the importance of cellular context when studying regulation of cancer.

In summary, gene-centric and network-aware analysis of CRC cell lines with AURKA knockdown shows that 20 Wnt and Ras-MAPK signalling genes, occurring in connected PPI networks in two CRC cell lines, are significantly deregulated by AURKA. The responses in the Wnt and Ras-MAPK pathways were different in the two cell lines; only KRAS (Ras-MAPK) and $\beta$-catenin (Wnt) were deregulated in both. Further, in both cell lines four genes connected AURKA to the deregulated genes in the Wnt and Ras-MAPK pathways: CDK6, UBE2D1, DICER 1 and RACGAP1. These results suggest possible genes and mechanisms for the interplay between AURKA and Wnt and Ras-MAPK signalling that are at the same time generic and unique between different CRC settings. Further investigation of the importance of Wnt and Ras-MAPK enhanced signalling by AURKA in CRC and the role of these genes for these interactions, will lead to a better understanding of the molecular mechanisms underlying CRC progression.

\section{Materials and Methods}

Cell culture and transfection with small interfering RNA (siRNA). SW480 cells were grown in Dulbecco's modified Eagle's medium (DMEM; Lonza, Verviers, Belgium) containing 10\% fetal bovine serum (FBS) (HyClone; Perbio Science, Etten-Leur, The Netherlands), and Caco 2 cells were grown in RPMI 1640 (Lonza) containing 20\% FBS. Both cell culture media were supplemented with $2 \mathrm{mM} \mathrm{L}$-glutamine, $100 \mathrm{IU} / \mathrm{ml}$ sodium penicillin (Astellas Pharma B.V., Leiderdorp, the Netherlands), and $100 \mathrm{mg} / \mathrm{ml} \mathrm{streptomycin}$ (Fisiopharma, Palomonta (SA), Italy). Transfection with siRNA pools (SMARTpools) from Dharmacon (Lafayette, Colorado, USA) was performed $24 \mathrm{~h}$ after seeding according to the manufacturer's recommendations. A final siRNA concentration of $30 \mathrm{nM}$ was obtained using DharmaFECT3 reagent (1:1000 dilution) for both cell lines. A non-targeting control siRNA pool (Non-Targeting Pool 2; D-001206-14) was used as a negative control.

RNA isolation. Total RNA was isolated using TriZol reagent (Invitrogen, Breda, the Netherlands) and subjected to purification using RNeasy Kit (Qiagen, Hilden, Germany). RNA concentrations and purities were measured on a Nanodrop ND-1000 spectrophotometer (Isogen, IJsselstein, the Netherlands). RNA quality was evaluated by generating an electropherogram on the Agilent Bioanalyzer 2100 using a RNA 6000 Nano-LabChip (Agilent Technologies, Santa Clara, CA, the Netherland). RNA integrity numbers (RIN) of $>9.0$ were considered as good quality RNA.

Microarray expression analyses. Microarray expression experiments were performed on $4 \times 44 \mathrm{~K}$ Agilent expression arrays (Agilent 60-mer SurePrint technologies), as described before ${ }^{30}$. Two biological replicates were performed for each siRNA experiment (both AURKA and non-targeting). Pre-processing and 
differential expression analysis (DEA) was done using the R-Bioconductor packages Limma ${ }^{31}$ and SVA-combat ${ }^{32}$. In short, conventional background correction was applied, followed by within-array normalization using Loess. Subsequently, batch-effect removal was applied for the replicate experiments, after which between-array normalization was done using the quantile method. The microarray data have been submitted to NCBI's Gene Expression Omnibus (GEO) and are accessible through GEO Series accession number GSE108320. DEA between AURKA siRNA transfected cells and non-targeted siRNA control transfected cells was assessed by using linear modelling and empirical Bayes statistics. Finally, p-values were adjusted for multiple testing using conventional Benjamini Hochberg FDR correction. The threshold for significantly expressed genes was set at a q-value less than 0.05 and a fold change greater than 1.5 or less than -1.5. The significantly deregulated genes are available in Supplementary Table S1 for SW480 and Supplementary Table S2 for Caco2. GO enrichment analysis was performed using the 'Gene Ontology enRIchment anaLysis and visuaLizAtion tool' (http://cbl-gorilla.cs.technion.ac.il/).

Probe ID mapping. The Agilent probe ids from the microarray expression experiments were mapped to their respective Ensembl ids and HGNC ids (when available) using BioMart mappings ('Agilent WholeGenome $4 \times 44 \mathrm{k}$ v1 probe') downloaded from www.ensembl.org/biomart/martview/ (January 2017). Additional mapping of HGNC ids to Ensembl ids was done using mappings from the Agilent website http://www.chem.agilent.com/ cag/bsp/gene_lists.asp (Human Genome, Whole - Four-Plex, 44 K) (January 2017). We manually checked and mapped the significantly expressed genes for each cell line. Out of the 30,889 and 30,105 Agilent probe ids 8,270 and 8,149 could not be mapped to Ensembl ids for Caco2 and SW480, respectively. Where the same Ensembl id was mapped to multiple Agilent probe ids, the Agilent probe id with the lowest p-value was chosen. This filtering step resulted in 24,589 and 24,088 probes whereof 16,319 and 15,939 has unique Ensembl ids for Caco2 and SW480, respectively.

Protein-protein interaction network. The Homo sapiens protein-protein interaction (PPI) network used in this analysis was retrieved from the STRING database (medium confidence: 0.400$)^{27}$ (downloaded: January 2017). The proteins were mapped to Ensembl ids using BioMart mappings (as described in the previous section). Self- and duplicate interactions were removed. The network consisted of 680,790 PPIs.

To describe the nodes (genes) and edges (interactions), one node-file and one edge-file were constructed for each cell line, SW480 and Caco2, based on the intersection of nodes (Ensembl ids) in the AURKA knockdown DEA data and the STRING PPI data. The edges-files consisted of two columns with Ensembl ids representing the two interacting proteins. The node-file consisted of three columns: nodes (Ensembl id), p-values, and $\log _{2}$ fold change. The Caco 2 edge-file consisted of 456,552 edges, and the SW480 edge-file consisted of 437,865 edges. The Caco2 node-file consisted of 12,249 nodes, while that for SW480 consisted of 12,082 nodes.

Identification of the most significantly deregulated gene modules. The Heinz algorithm ${ }^{26}$ was applied to determine the most significantly deregulated gene module (sets of genes connected in the PPI network) for each cell line, SW480 and Caco2, using the constructed edge- and node-files. Each node in the network was assigned a weight reflecting its p-value from the DEA. A beta-uniform mixture model was fitted to the p-value distribution determining its parameters: the shape parameter, $\alpha$, and the mixture parameter, $\lambda^{33}$. The $\alpha$ parameter was 0.342 for SW480 and 0.329 for Caco2. Due to the high amount of low p-values, the $\lambda$ parameter was approximated 0.0 in both cell lines. Before calculating the weights of the nodes we set the $\lambda$ parameters to 0.1 , which still gave a very reasonable fit. The Heinz algorithm then assigned weights to the nodes using the $\lambda$ and $\alpha$ parameters, the p-values and a false-discovery rate (FDR) threshold. Positive weights were given to nodes with a p-value lower than the FDR threshold, whereas negative weights were given to nodes with a p-value greater than the FDR threshold. The FDR threshold for SW480 was $6.21 \mathrm{e}-4$ corresponding to a p-value of $4.46 \mathrm{e}-5$. The FDR threshold for $\mathrm{Caco} 2$ was $2.40 \mathrm{e}-4$ corresponding to a p-value of $1.37 \mathrm{e}-5$. The Heinz algorithm subsequently calculated the maximum-scoring subnetwork of the STRING PPI network that represents the most significantly deregulated gene module. The genes in these modules and their $\mathrm{p}$-values and $\log _{2}$ fold changes are available in Supplementary Table S3 for SW480 and Supplementary Table S4 for Caco2.

Since the FDR threshold has an influence on how many nodes are assigned positive weights, it also has an influence on the size of the significantly deregulated gene module. We selected the FDR thresholds so that 50 genes had a positive weight for each cell line. These thresholds are a conservative choice to generate focus on the part of the network that shows the strongest deregulation. This means that genes with p-values just above the threshold may still be significantly deregulated. The resulting modules selected by Heinz are not very sensitive to the FDR setting, simply said the modules just incrementally grow or shrink, as the threshold is adapted.

Determining Wnt and Ras-MAPK signalling genes. To define which genes are in the Wnt and Ras-MAPK pathways we downloaded the gene lists of the 'Wnt signalling pathway' 'MAPK signalling pathway' and 'Ras signalling pathway' from the KEGG database (January 2017) ${ }^{34}$. These genes were used to annotate Wnt and Ras-MAPK signalling genes in the results.

Shortest path analysis. To find possible connections between AURKA and the signalling pathways of interest, we performed a shortest-path analysis. The shortest path is defined as the minimum number of edges required to travel from one node in the PPI graph to another. First, we reduced the STRING PPI network to a subnetwork only consisting of the significantly deregulated genes determined by the DEA and the genes located in the most significantly deregulated gene module in the Heinz analysis. We then applied the Python package NetworkX to compute all the shortest paths between AURKA and the Wnt and Ras-MAPK signalling genes, where the edges were unweighted. 
TCGA CRC data analysis. To compare the effect of AURKA on Wnt and Ras-MAPK signalling in CRC tissue data to our CRC cell line results we analysed the TCGA COADREAD ${ }^{5}$ RNA-seq and somatic copy number aberration data for 330 microsatellite stable tumour samples retrieved from Firehose (March 2017). First, the samples were labelled by AURKA-gain and AURKA-no-gain based on the copy number aberration data in the genome region 54,944,445-54,967,393 (genome build 19) encoding the AURKA gene. Samples with a segment mean greater than or equal to 0.4 were labelled $A U R K A$-gain, whereas samples with a segment mean less than 0.4 were labelled AURKA-no-gain (Supplementary Table S5). The segment mean is the average of the tumour versus normal intensity $\log _{2}$ ratio, thereby describing the copy number aberrations. We selected the threshold of 0.4 following the assumption that the gain of AURKA also increases its expression (Supplementary Fig. S2). Second, DEA was performed between the AURKA-no-gain and AURKA-gain samples. The atypical choice of performing a no-gain vs. gain comparison (instead of gain vs. no-gain) is because of the distinctive setup of the cell line experiments themselves, where the non-perturbed cell lines have AURKA gain and the perturbed cell lines have an AURKA downmodulation (no-gain). Significantly expressed genes based on the DEA were determined at an adjusted p-value less than $10^{-5}$ (Supplementary Fig. S3). The significantly deregulated genes are available in Supplementary Table S6. We then investigated the significance and directionality of the genes located on 20q. First, we retrieved the Ensembl ids for the genes located on 20q11-20q13.33 from BioMart. Second, we mapped these Ensembl ids to the Ensembl ids in the TCGA samples. The TCGA DEA result for these genes is available in Supplementary Table S7. Finally, we determined the overlap of significantly deregulated genes between the TCGA DEA and the DEA of SW480 and Caco2 (Supplementary Fig. S4).

\section{References}

1. Ferlay, J. et al. Cancer incidence and mortality worldwide: sources, methods and major patterns in GLOBOCAN 2012. Int J Cancer 136, E359-E386 (2015)

2. Hanahan, D. \& Weinberg, R. A. Hallmarks of cancer: the next generation. Cell 144, 646-674 (2011).

3. Fearon, E. R. Molecular genetics of colorectal cancer. Annu Rev Pathol 6, 479-507 (2011).

4. Haan, J. C. et al. Genomic landscape of metastatic colorectal cancer. Nat Commun 5, 5457 (2014).

5. The Cancer Genome Atlas Network. Comprehensive molecular characterization of human colon and rectal cancer. Nature 487, 330-337 (2012)

6. Matano, M. et al. Modeling colorectal cancer using CRISPR-Cas9-mediated engineering of human intestinal organoids. Nature Medicine 21, 256-262 (2015).

7. Lengauer, C., Kinzler, K. W. \& Vogelstein, B. Genetic instability in colorectal cancers. Nature 386, 623-627 (1997).

8. Meijer, G. A. et al. Progression from colorectal adenoma to carcinoma is associated with non-random chromosomal gains as detected by comparative genomic hybridisation. J Clin Pathol 51, 901-909 (1998).

9. Nakao, K. et al. High-resolution analysis of DNA copy number alterations in colorectal cancer by array-based comparative genomic hybridization. Carcinogenesis 25, 1345-1357 (2004).

10. Carvalho, B. et al. Multiple putative oncogenes at the chromosome $20 \mathrm{q}$ amplicon contribute to colorectal adenoma to carcinoma progression. Gut 58, 79-89 (2009).

11. Sillars-Hardebol, A. H. et al. TPX2 and AURKA promote $20 \mathrm{q}$ amplicon-driven colorectal adenoma to carcinoma progression. Gut 61, 1568-1575 (2012)

12. Tanner, M. M. et al. Amplification of Chromosomal Region 20q13 in Invasive Breast Cancer: Prognostic Implications. Clin Cancer Res 1, 1455-1461 (1995).

13. Aust, D. E. et al. Prognostic relevance of $20 \mathrm{q} 13$ gains in sporadic colorectal cancers: a FISH analysis. Scand J Gastroenterol 39, 766-772 (2004)

14. Lassus, H., Staff, S., Leminen, A., Isola, J. \& Butzow, R. Aurora-A overexpression and aneuploidy predict poor outcome in serous ovarian carcinoma. Gynecol Oncol 120, 11-17 (2011).

15. Belt, E. J. et al. High lymph node yield is related to microsatellite instability in colon cancer. Ann Surg Oncol 19, 1222-1230 (2012).

16. Goos, J. A. C. M. et al. Aurora kinase A (AURKA) expression in colorectal cancer liver metastasis is associated with poor prognosis. Br J Cancer 109, 2445-2452 (2013).

17. Zeng, B. et al. Aurora-A is a novel predictor of poor prognosis in patients with resected lung adenocarcinoma. Chin J Cancer Res 26, 166-173 (2014).

18. Dar, A. A., Belkhiri, A. \& El-Rifai, W. The aurora kinase A regulates GSK-3 $\beta$ in gastric cancer cells. Oncogene 28, 866-875 (2009).

19. Liu, X. et al. AURKA induces EMT by regulating histone modi cation through Wnt/ $\beta$-catenin and PI3K/Akt signaling pathway in gastric cancer. Oncotarget 7, 33152-33164 (2016).

20. Xia, Z. et al. AURKA governs self-renewal capacity in glioma-initiating cells via stabilization/activation of $\beta$-catenin/Wnt signaling. Mol Cancer Res 11, 1101-1111 (2013).

21. Umstead, M., Xiong, J., Qi, Q., Du, Y. \& Fu, H. Aurora kinase A interacts with H-Ras and potentiates Ras-MAPK signaling. Oncotarget 8, 28359-28372 (2017).

22. Furukawa, T. et al. AURKA is one of the downstream targets of MAPK1/ERK2 in pancreatic cancer. Oncogene 25, 4831-4839 (2006).

23. Dutta-Simmons, J. et al. Aurora kinase A is a target of Wnt/ $\beta$-catenin involved in multiple myeloma disease progression. Blood 114, 2699-2708 (2009).

24. Jacobsen, A. et al. Construction and Experimental Validation of a Petri Net Model of Wnt/3-Catenin Signaling. PLoS One 11, e0155743, https://doi.org/10.1371/journal.pone.0155743 (2016).

25. Ahmed, D. et al. Epigenetic and genetic features of 24 colon cancer cell lines. Oncogenesis 2, e71 (2013).

26. Dittrich, M. T., Klau, G. W., Rosenwald, A., Dandekar, T. \& Muller, T. Identifying functional modules in protein-protein interaction networks: an integrated exact approach. Bioinformatics 24, i223-i231 (2008).

27. Szklarczyk, D. et al. STRINGv10: protein-protein interaction networks, integrated over the tree of life. Nucleic Acids Res 43, D447-D452 (2015).

28. Guinney, J. et al. The consensus molecular subtypes of colorectal cancer. Nat Med 21, 1350-1356 (2015).

29. Ubink, I. et al. A Novel Diagnostic Tool for Selecting Patients With Mesenchymal-Type Colon Cancer Reveals Intratumor Subtype Heterogeneity. J Natl Cancer Inst 109, djw303 (2017).

30. Buffart, T. E. et al. NMD inhibition fails to identify tumour suppressor genes in microsatellite stable gastric cancer cell lines. BMC Med Genomics 2, 39 (2009).

31. Ritchie, M. E. et al. limma powers differential expression analyses for RNA-sequencing and microarray studies. Nucleic Acids Res 43 , e47 (2015).

32. Leek, J. T. \& Storey, J. D. Capturing Heterogeneity in Gene Expression Studies by Surrogate Variable Analysis. PLoS Genetics 3, e161 (2007). 
33. Beisser, D., Klau, G. W., Dandekar, T., Müller, T. \& Dittrich, M. T. BioNet: an R-Package for the functional analysis of biological networks. Bioinformatics 26, 1129-1130 (2010).

34. Kanehisa, M., Furumichi, M., Tanabe, M., Sato, Y. \& Morishima, K. KEGG: new perspectives on genomes, pathways, diseases and drugs. Nucleic Acids Res 45, D353-D361 (2017).

\section{Acknowledgements}

This study was supported by National Institute for Health Research (NIHR) Biomedical Research Centre based at Guy's and St Thomas' NHS Foundation Trust and King's College London, Netherlands Organisation for Scientific Research (NWO grant 612.001.203), and Dutch Cancer Society (KWF Fellowship 2013-5885).

\section{Author Contributions}

Designed the experiments and provided advice: A.J., L.J.W.B., S.R.M.d.K., B.C., A.H.S.H., R.J.D., E.d.R., G.A.M., S.A., J.H., R.J.A.F., K.A.F. Performed the experiments and analyzed the data: A.J., L.J.W.B. and A.H.S.H. Wrote the paper: A.J., L.J.W.B., S.R.M.d.K., B.C., S.A., J.H., R.J.A.F. and K.A.F. All authors reviewed the manuscript.

\section{Additional Information}

Supplementary information accompanies this paper at https://doi.org/10.1038/s41598-018-24982-z.

Competing Interests: The authors declare no competing interests.

Publisher's note: Springer Nature remains neutral with regard to jurisdictional claims in published maps and institutional affiliations.

(c) (i) Open Access This article is licensed under a Creative Commons Attribution 4.0 International License, which permits use, sharing, adaptation, distribution and reproduction in any medium or format, as long as you give appropriate credit to the original author(s) and the source, provide a link to the Creative Commons license, and indicate if changes were made. The images or other third party material in this article are included in the article's Creative Commons license, unless indicated otherwise in a credit line to the material. If material is not included in the article's Creative Commons license and your intended use is not permitted by statutory regulation or exceeds the permitted use, you will need to obtain permission directly from the copyright holder. To view a copy of this license, visit http://creativecommons.org/licenses/by/4.0/.

(C) The Author(s) 2018 